\title{
REVISÃO CRÍTICA DO OPM3: UM ESTUDO DE REDUNDÂNCIAS
}

\section{OPM3 CRITICAL REVIEW: A STUDY TOWARD REDUNDANCY}

\author{
Nemer Alberto Zaguir ${ }^{1}$; Marcelo Ramos Martins ${ }^{2}$ \\ ${ }^{1}$ Escola Politécnica da Universidade de São Paulo - Brasil nemer.zaguir@oracle.com \\ ${ }^{2}$ Escola Politécnica da Universidade de São Paulo - Brasil mrmartin@usp.br
}

\begin{abstract}
Resumo
As questões de ética e responsabilidade social são temas cada vez mais presentes nas estratégias organizacionais. Os mecanismos de gestão que visam estabelecer vínculos entre estratégia e operação contribuem para a incorporação destes temas na cultura corporativa. O OPM3, modelo de maturidade organizacional em gerenciamento de projetos, tem como missão suportar as organizações a desenvolverem capacidades para alinhar seus objetivos estratégicos com sua operação através de projetos. Apesar de seu desenvolvimento ter sido fundamentado em diversas pesquisas e com a contribuição de mais de 700 especialistas, não está isento de críticas. O objetivo deste trabalho é apresentar críticas existentes e desenvolver outras relacionadas à redundância do modelo. Neste artigo, a investigação da redundância é tratada pela técnica de modelagem de dados $e$ as apresentadas como já existentes obtidas na literatura. O resultado da investigação indica como transformar 456 melhores práticas, dentre as 586 apresentadas pelo OPM3, em 38 melhores práticas típicas e 120 perguntas, dentre as 151 do questionário preliminar, em 10 perguntas estruturadas. Conclui-se o trabalho apresentando as vantagens das críticas para aqueles que pretendem aprimorar a maturidade em gerenciamento de projetos de sua organização, pesquisadores da área de maturidade e revisores do OPM3.
\end{abstract}

Palavras-chave: modelo de maturidade em gerenciamento de projetos; críticas ao OPM3; vínculo entre estratégia e operação.

\section{Introdução}

O gerenciamento de projetos pode ser visto como uma disciplina que visa não só melhoria nas condições econômicas de uma firma, mas como um conjunto de práticas geralmente aceitas que estimulam governança, transparência e que podem fortalecer os vínculos entre estratégia e operação. Neste sentido, os temas ética e responsabilidade social, quando presentes em uma estratégia, podem ser disseminados através dos mecanismos de gestão de projetos. Assim, melhorias e amadurecimento nesses sistemas contribui para que diretrizes, políticas e práticas de projetos sejam cada vez mais relevantes para o exercício de valores como ética em uma cultura e 
para a consideração efetiva da sociedade e seus anseios como parte interessada que influencia e é influenciada pelos resultados dos projetos.

O Organizational Project Management Maturity Model (OPM3), modelo de maturidade organizacional para gestão de projetos elaborado pelo PMI, Project Management Institute (PMI, 2003 ) entre 1998 e 2003, tem como missão suportar as organizações a desenvolverem capacidades necessárias a fim de alinhar seus objetivos estratégicos com a sua operação através de projetos. Desta forma, este modelo pode ser visto como um dos sistemas para apoiar a disseminação de valores e iniciativas da estratégia organizacional.

O OPM3 foi concebido e testado a partir de diversas pesquisas, utilização de técnicas da gestão da qualidade, como o "Quality Function Deployment" e "House of Quality" para coletar e testar requisitos dos usuários (PMI, 2003), forte participação de reconhecidos especialistas da área de gerenciamento de projetos e patrocínio executivo do PMI. Apesar da tentativa em buscar sintonia do produto com necessidades dos setores industriais representados por mais de 700 voluntários, o OPM3 não está isento de críticas quanto a sua complexidade e aplicabilidade.

Este trabalho foi motivado pela iniciativa do primeiro autor em aplicar o OPM3 em uma empresa fornecedora de serviços de Tecnologia da Informação (TI) onde teve a oportunidade de olhar de forma crítica sua estrutura e conteúdo. O trabalho tem como objetivo investigar críticas existentes ao OPM3 e redundâncias em sua estrutura que poderão servir para uma futura revisão do modelo.

A próxima seção traz a metodologia utilizada e a terceira apresenta uma revisão bibliográfica em três partes: um apanhado sobre modelos de maturidade até o surgimento do OPM3, um resumo do modelo e as críticas existentes. A quarta seção apresenta os resultados da investigação de redundâncias na estrutura do OPM3 e por último, discute-se os resultados e são indicados trabalhos futuros relevantes para continuidade da pesquisa.

A visão crítica apresentada beneficia pesquisadores da área de maturidade em gerenciamento de projetos, interessados em avaliar a maturidade da prática de gestão de projetos em suas organizações e poderá servir como fonte para a revisão do OPM3 em andamento pelo PMI.

\section{Metodologia}

O trabalho foi realizado em duas etapas: a primeira consistiu em realizar uma revisão na literatura para identificar as críticas existentes ao OPM3 e a segunda em identificar as redundâncias na estrutura do modelo.

Na segunda etapa foi utilizada a técnica de modelagem de dados, proveniente da área de Tecnologia da Informação. Segundo Setzer \& Silva (2005), a estrutura de um sistema pode ser 
analisada segundo vários níveis de abstrações. O nível descritivo, das descrições informais pode ser representado através de uma linguagem natural. O nível dos dados conceituais pode ser representado através do modelo entidade-relacionamentos (MER). Este modelo utiliza o formalismo da teoria dos conjuntos e um diagrama para representar a estrutura de dados de determinada realidade. Durante o desenvolvimento do trabalho foi elaborado o MER da atual estrutura do OPM3 que serviu como ferramenta para facilitar a compreensão das interdependências das relações entre os seus elementos e na identificação de redundâncias em sua estrutura.

\section{Revisão bibliográfica - modelos de maturidade e o OPM3}

A noção de melhoria em processos e conseqüente benefícios para o negócio foi amplamente disseminada no movimento de gestão da qualidade total com Deming (1982) através das técnicas de controle estatístico de processos. Alguns modelos de maturidade de processos de negócios da gestão da qualidade foram utilizados para levantar a maturidade da gestão de projetos, tais como o Baldridge National Quality Award e o Business Excellence Model do European Forum for Quality Management (COOKE-DAVIES et al.,2001). Humphrey (1989) extrapolou o conceito de maturidade de processos da gestão da qualidade para a medida de maturidade "organizacional", estabelecendo escala de maturidade em 5 níveis, utilizados para classificar cada processo de desenvolvimento de software baseado no desenvolvimento de capacidades. O modelo explicita quais capacidades devem ser desenvolvidas para que um processo classificado em determinado nível de maturidade possa atingir o próximo nível. Com fundamentação no trabalho de Humphrey (1989), o Software Engineering Institute (SEI) da Carnegie-Mellon University lança em 1993 o Capability Maturity Model (CMM) e em 2002 o Capability Maturity Model Integrated (CMM-I) (CMM-I, 2002). Após o lançamento do CMM começam a surgir outros modelos de maturidade em gerenciamento de projetos (Morris, 2000).

Em 1996 o PMI publica uma nova versão do PMBoK (Project Management Body of Knowledge) (PMI, 2004), proliferado internacionalmente e cresce o número de gerentes de projetos com a certificação PMP (Project Manager Professional). Com o PMBoK e este processo de certificação, o PMI cobre o desenvolvimento e práticas de gerenciamento de projetos no âmbito do projeto e no da profissionalização ou do indivíduo, mas percebe uma clara lacuna para este desenvolvimento no âmbito da organização. Em maio de 1998 o PMI lança um programa para desenvolver um padrão para ajudar as organizações a levantar e melhorar as suas capacidades em gestão de projetos e realizar sua estratégia organizacional, dando início ao desenvolvimento do

OPM3. Em 1999, J.Schlichter, diretor do programa, lançou suas prioridades estratégicas (SCHLICHTER et al., 2003) e deu-se inicio a uma pesquisa para verificar se já existia um modelo 
de maturidade suficiente para medir a maturidade organizacional de gestão de projetos. Esta pesquisa tomou 27 modelos de maturidade do mercado e concluiu que não havia nenhum adequado para o propósito do OPM3 (COOKE-DAVIES et al., 2001). O OPM3 desenvolvido é lançado pelo PMI no final de 2003.

O programa OPM3 é constituído por um conjunto de 3 elementos: "conhecimento", "levantamento" e "plano de melhoria". O elemento "conhecimento", denominado standard contém a descrição do modelo e a forma de aplicá-lo. O elemento "levantamento" apresenta os passos e ferramentas para sua aplicação. O "plano de melhoria" é um guia do método que permite planejar e priorizar o desenvolvimento de capacidades e implementação de melhores práticas.

A estrutura do OPM3 é concebida em 4 dimensões: A primeira trata o relacionamento de uma melhor prática, 586 disponíveis, com um conjunto de capacidades que necessitam estar desenvolvidas para o que, segundo o padrão, esta melhor prática seja reconhecida como implementada. A segunda estabelece o relacionamento entre uma melhor prática e suas capacidades a um domínio, que pode ser o projeto, o programa (conjunto de projetos) ou portifólio (conjunto de todos os programas e projetos da Organização). O termo “domínio_PPP” será utilizado no texto para representar o conjunto com estes 3 elementos. A terceira dimensão estabelece o relacionamento de uma melhor prática e suas capacidades a um determinado estágio de maturidade de processo: estágio de padronização (standard), medição (measured), controle (controlled) e o estágio da melhoria contínua (improvement). O termo "estágio_SMCI" representa o nome deste conjunto no texto. A quarta dimensão refere-se à associação de uma capacidade a determinado grupo de processo de gestão, conceito extraído do PMBoK (PMI, 2004), a saber: inicialização (initialization), planejamento (planning), execução (execution), controle (controlling) e encerramento (closing). O termo "grupo de processos IPECC" representa o nome deste conjunto no texto.

O modelo indica 5 passos para sua utilização. O primeiro recomenda à equipe que vai utilizá-lo conhecer o standard. O segundo, denominado levantamento, é subdividido em duas etapas: o levantamento de alto nível ou preliminar e o levantamento compreensivo ou de capacidades. O levantamento de alto nível consiste de um questionário com 151 perguntas utilizado na produção de 4 gráficos que indicam, de forma geral, a maturidade da organização frente ao padrão. Após este levantamento, a empresa pode iniciar a segunda etapa do levantamento com enfoque no estudo das capacidades. O terceiro passo consiste na elaboração de um plano de melhoria com base nas melhores práticas e capacidades identificadas como não desenvolvidas no âmbito do domínio e estágio de maturidade de interesse. O quarto e quinto passos são respectivamente recomendações para a implementação deste plano e repetição do processo visando sua melhoria contínua. 


\section{Atuais críticas ao OPM3 obtidas na literatura}

Este tópico destaca quatro críticas ou comentário diretos ao OPM3 pesquisados na literatura:

a) Kenneth (2004) comenta que com a publicação do OPM3, o PMI completou o tripé que suporta a implementação da gestão de projetos nas dimensões do projeto, dos indivíduos e da organização. A dimensão de projetos já estava coberta pela publicação do $P M B o K$ guide (PMI,2004) e a dos "indivíduos", pela publicação do "Project Management Competency Development Framework". Mostra que o OPM3 é um passo significante para o avanço da disciplina de gestão de projetos, mas apesar de ser um "produto" deve continuar evoluindo na medida que a profissão de gerente de projetos continua crescendo. Recomenda que os usuários do OPM3 não podem iniciar a aplicação do OPM3 a partir das ferramentas e esperar resultado satisfatório. Devem aprofundar-se no conhecimento do standard;

b) Alleman (2006) questiona se a comunidade de gerentes de projetos realmente precisa do OPM3 uma vez que o modelo SEI-CMM-I (CMM-I, 2002) apresenta 9 sub-sessões voltadas especialmente para a gestão de projetos e ainda considera de forma importante os aspectos técnicos e do negócio em que se insere a prática de gestão de projetos. Argumenta que a gestão de projetos é um processo habilitador e não estratégico. Como exemplo, cita que durante um projeto, o processo de gestão do projeto é sempre executado como um processo secundário frente aos processos que conduzem ao produto final esperado. Questiona o fato do OPM3 ter 586 melhores práticas. Apresenta como exemplos 0 "Software Program Managers Network" (http://www.spmn.com/16CSP.html) com 16 melhores práticas e o "Project Breathalyzer". Coloca que o OPM3 deveria ser separado em três partes pela natureza distinta de suas atividades: a primeira deveria tratar aspectos "organizacionais", a segunda "gestão projetos" e a terceira "modelo de maturidade". Para tratar a parte “organizacional” propõe que seja utilizado o "Balanced Scorecard”, método reconhecido para definir, dirigir e levantar estratégia de negócios e alinhá-la com as ações na operação. Para tratar "gestão de projetos", propõe a utilização de um "project management handbook", tal como CMM-I ou PMBoK e para a questão da maturidade de processos, propõe a aplicação do próprio CMM-I;

c) Soler (2005) apresenta o OPM3 como sendo uma trilha segura e referenciada capaz de orientar gestores organizacionais em seus investimentos na área de projetos, sendo um modo eficaz de propor mudanças destinadas à melhoria da gestão de projetos. 
Destacamos 4 críticas diretas: a) dificuldade de entendimento dos usuários do OPM3 em função do OPM3 não estar traduzido para o Português; b) interpretação das melhores práticas para os domínios do programa e portifólio repetitiva e desprovida de significado. Justifica esta crítica a ausência de um guia de referência para processos no domínio de programa e de portifólio; c) questionário de avaliação considerado repetitivo e burocrático; d) ausência de um grau mensurável associado à avaliação de maturidade, tal como estabelecem outros modelos de maturidade, dificulta o entendimento, a comunicação interna e o estabelecimento de metas mensuráveis e compromissos para o aprimoramento da maturidade organizacional através do OPM3;

d) Hillson (2003), em seu trabalho sobre capacidades organizacionais em gestão de projetos publicado no "Journal of Facilities Management" apresenta o "The Project Management Maturity Model (ProMMM)". Neste trabalho, estimula a aplicação do ProMMM para a área de gestão de projetos em instalações industriais e comenta que provavelmente potenciais usuários tem sido fortemente desencorajados em aplicar o OPM3 pela grande abrangência de seu escopo, estrutura extremamente complexa. O trabalho não apresenta nenhum estudo ou fundamentação para demonstrar o argumento.

\section{Resultados da Identificação de Redundâncias}

Redundância no diretório de melhores práticas:

O MER do OPM3 utilizado no desenvolvimento do trabalho facilitou a investigação de uma restrição imposta pelo modelo: uma melhor prática está associada a um e somente uma única combinação de domínio_PPP e estágio de maturidade SMCI (a melhor prática número "1000” é a única exceção). Se for necessário que uma melhor prática seja representada em todos os domínios em todos os estágios, não podemos, segundo o modelo, utilizar uma única melhor prática, mas sim 12, produto cartesiano entre o conjunto “domínio PPP” e “estágio SMCI”. A tabela 1 ilustra 6 casos do mesmo tipo de melhor prática. Apesar de não ter estar na tabela, existem ainda mais 6 melhores práticas que compartilham a raiz "Plan Development". 
Tabela 1 - Exemplo do diretório de melhores práticas do OPM3

\begin{tabular}{|c|c|c|c|c|c|c|c|c|c|c|}
\hline BP ID & \multicolumn{2}{|l|}{ Title } & Description & \multirow{2}{*}{$\frac{\mathbf{P}}{\mathrm{X}}$} & \multirow[t]{2}{*}{$\mathbf{P}$} & \multirow[t]{2}{*}{$\mathbf{P}$} & \multirow{2}{*}{$\frac{\mathbf{S}}{\mathrm{X}}$} & \multirow[t]{2}{*}{$\mathbf{C}$} & \multirow[t]{2}{*}{$\mathbf{M}$} & \multirow[t]{2}{*}{ I } \\
\hline 1020 & $\begin{array}{l}\text { Project } \\
\text { Development } \\
\text { Standardization }\end{array}$ & $\begin{array}{r}\text { Plan } \\
\text { Process }\end{array}$ & $\begin{array}{l}\text { Project Plan Development process } \\
\text { standards are established }\end{array}$ & & & & & & & \\
\hline 3120 & $\begin{array}{l}\text { Program } \\
\text { Development } \\
\text { Standardization }\end{array}$ & $\begin{array}{r}\text { Plan } \\
\text { Process }\end{array}$ & $\begin{array}{l}\text { Program Plan Development process } \\
\text { standards are established }\end{array}$ & & $\mathrm{X}$ & & $\mathrm{X}$ & & & \\
\hline 4790 & $\begin{array}{l}\text { Portifolio } \\
\text { Develoment } \\
\text { Standardization }\end{array}$ & $\begin{array}{r}\text { Plan } \\
\text { Process }\end{array}$ & $\begin{array}{l}\text { Portifolio Plan Development process } \\
\text { standards are established }\end{array}$ & & & $\mathrm{X}$ & $\mathrm{X}$ & & & \\
\hline 1710 & $\begin{array}{l}\text { Project } \\
\text { Development } \\
\text { Measurement }\end{array}$ & $\begin{array}{r}\text { Plan } \\
\text { Process }\end{array}$ & $\begin{array}{l}\text { Project Plan Development Process } \\
\text { measures are established, assembled } \\
\text { and analysed }\end{array}$ & $\mathrm{X}$ & & & & $\mathrm{X}$ & & \\
\hline 2250 & $\begin{array}{l}\text { Project } \\
\text { Development } \\
\text { Control }\end{array}$ & $\begin{array}{r}\text { Plan } \\
\text { Process }\end{array}$ & $\begin{array}{l}\text { Project Plan Development Process } \\
\text { controls are established and executed } \\
\text { to control the stability of the process }\end{array}$ & $\mathrm{X}$ & & & & & $\mathrm{X}$ & \\
\hline 2640 & $\begin{array}{l}\text { Project } \\
\text { Development } \\
\text { Improvement }\end{array}$ & $\begin{array}{r}\text { Plan } \\
\text { Process }\end{array}$ & $\begin{array}{l}\text { Project Plan Development Process } \\
\text { problem areas are assessed, process } \\
\text { improvement recomendations are } \\
\text { collected and process improvement } \\
\text { are implemented }\end{array}$ & $\mathrm{X}$ & & & & & & $\mathrm{X}$ \\
\hline
\end{tabular}

Fonte: Adaptado de Project Management Institute: Organisational Project Management Maturity Model - OPM3 (PMI, 2003)

Se introduzirmos o conceito de, "melhor prática típica", forçando a eliminação da restrição mencionada e permitindo a independência na identificação da "melhor prática" do domínio PPP e estágio SMCI teríamos ao invés de 12 melhores práticas, apenas 1 "melhor prática típica", suficiente para cobrir o mesmo conteúdo de análise de maturidade, conforme tabela 2.

Tabela 2 - Exemplo de Melhor Prática Típica

\begin{tabular}{|c|c|c|}
\hline BP_ID & \multicolumn{1}{|c|}{ Title } & Description \\
\hline & $\begin{array}{l}\text { Desenvolvimento do Plano de } \\
\text { trabalho }\end{array}$ & $\begin{array}{c}\text { Desenvolvimento do plano de trabalho (relevante para todos as } \\
\text { combinações de domínios e estágios de maturidade) }\end{array}$ \\
\hline
\end{tabular}

Fonte: Elaborado pelos autores

Deve-se observar que podem existir melhores práticas específicas para determinadas combinações de domínio e estágio onde não faz sentido a sua combinação com todos os elementos do produto cartesiano entre domínio e estágio. Estas melhores práticas não podem portanto serem reduzidas ao conceito de melhor prática típica. Em uma revisão do modelo, deve-se obsservar a existência destas melhores práticas. Se realmente existirem, não se pode eliminar o atual conceito atual de melhor prática do modelo.

Redundância nas 151 perguntas do levantamento preliminar:

O levantamento de alto nível ou preliminar da primeira etapa do passo de "levantamento" consiste na resposta do tipo "sim" ou "não" a 151 perguntas de um questionário. Observamos que 
120 perguntas estão relacionadas diretamente a um tipo de agrupamento dos processos IPECC do PMBoK e a um agrupamento que classifica um processo como core (fundamental) ou de suporte, totalizando 10 grupos de perguntas ou perguntas básicas.

Para ilustrar, mostramos como identificar uma pergunta básica sobre os grupo de processos "planejamento" (P) que envolve os processos fundamentais. Deve-se observar que esta pergunta básica é apresentada no questionário doze vezes: quatro vezes para o domínio de projeto, uma para cada estágio de maturidade (perguntas 17, 32, 40 e 49), quatro vezes para o domínio de programa, uma para cada estágio de maturidade (perguntas $61,70,78,86$ ) e mais quatro vezes para o domínio de portifólio, uma para cada estágio de maturidade( perguntas 111,116, 134 e 145). Abaixo representamos apenas as perguntas 17,32, 40 e 49:

a) 17: Does your organization establish and use standard documented processes at the Project level for the Planning Core Processes (Project Plan Development, Scope Planning, Scope Definition, Activity Definition, Activity Sequencing, Activity Duration Estimating, Schedule Development, Resource Planning, Cost Estimating, Cost Budgeting, Risk Management Planning)? ( project, standard);

b) 32: Does your organization establish and use measurements at the Project level for the Planning Core Processes? (project, measured);

c) 40: Does your organization establish and execute controls at the Project level to manage the stability of Planning Core Processes? ( project, controlled);

d) 49: Does your organization identify, assess, and implement improvements at the Project level for the Planning Core Processes? (project, improvement);

A pergunta básica que representaria as doze perguntas pode ser estruturada da seguinte forma, coforme tabela 3: "Os processos fundamentais de planejamento apresentam-se em quais estágios de maturidade em cada domínio?”.

Tabela 3 - Complementação da pergunta básica

\begin{tabular}{|l|l|l|l|l|}
\hline & Padronizado (S) & Medido (M) & Controlado (C) & Melhoria (I) \\
\hline Projeto (P) & & & & \\
\hline Programa (P) & & & & \\
\hline Portifólio (P) & & & & \\
\hline
\end{tabular}

Fonte: Elaborado pelos autores

Pelo exemplo inferimos que o sistema de perguntas poderia ser melhor estruturado e otimizado. Ao invés de 120 perguntas, poderia-se ter 10 perguntas básicas colocadas de forma a cobrir o levantamento para a combinação dos domínios e estágios de maturidade, reduzindo o 
questionário sem nenhuma perda, além de nova estrutura poder facilitar a produção do diagnóstico e gráficos resultantes do questionário.

Uma observação crítica do questionário modificado poderia indicar que a síntese das perguntas não é positiva no sentido de que a redundância e distribuição das perguntas poderia existir para identificar algum tipo de incoerência nas respostas dos entrevistados. Deve-se notar que se a resposta na tabela for "não" em determinada coluna de uma linha, obrigatoriamente será "não" para as demais colunas à direita desta mesma linha. Isto é válido uma vez que não faz sentido existir um processo que não está desenvolvido em determinado estágio de maturidade e estar em um estágio superior. Com a distribuição das perguntas poder-se-ia identificar respostas inconsistentes e avaliar a qualidade do levantamento. O OPM3 não provê ferramentas para avaliar este tipo de inconsistência.

\section{Conclusões}

O trabalho apresenta 4 grupos de críticas existentes por autor, sendo as principais relacionadas à redundância, complexidade e dificuldade de interpretação dos resultados da aplicação do modelo.

A investigação da redundância na estrutura do OPM3 permitiu constatar dois importantes resultados: o primeiro em relação a identificação de alta redundância no diretório de melhores práticas e a indicação para transformar 456 melhores práticas de 586 do modelo em 38 melhores práticas típicas, reduzindo o volume total do diretório para 168. O segundo, a identificação de redundância nas perguntas do questionário de alto nível e a conseqüente indicação para transformar 120 perguntas das 151 do questionário em 10 perguntas básicas mais estruturadas, reduzindo o volume de perguntas para 41, sem nenhuma perda de conteúdo.

Desta forma, a pesquisa confirma críticas existentes com relação ao aspecto de redundância do OPM3 e indica potencial melhoria direta na estrutura do modelo .

O trabalho não estudou redundâncias abrangendo o diretório de capacidades, não tratou críticas sobre o processo de aplicação do modelo e tampouco criticou o conteúdo das melhores práticas ou capacidades. Estes estudos poderiam ser realizados a fim de enriquecer a qualidade da revisão do modelo.

Foram identificados alguns artigos que tratam o tema sobre modelos de maturidade mas que não mencionam ou aplicam diretamente o OPM3. Estes artigos poderiam ser utilizados para levantar potenciais críticas ao modelo a serem confirmadas em outras pesquisas. Estes trabalhos são: Sommerville \& Ransom (2005); Sawyer et al (1999); Cooke-Davies \& Arzymanow (2003); 
Sommerville \& Langford (1992); Contruction Industry Institute (1993); Rabechini \& Carvalho (2003); Bouer \& Carvalho (2005); Jugdev \& Thomas (2002) e Kerzner (2001).

A visão crítica compartilhada pelo trabalho pode beneficiar diretamente pesquisadores na área de modelos de maturidade em gestão de projetos, aqueles interessados em avaliar a maturidade da prática de gestão de projetos em suas organizações e servir como fonte para futuras críticas e revisão do OPM3. Na qualidade de usuários potenciais do OPM3, os interessados em aprimorar o estudo sobre a tradução da estratégia em operação através de projetos e interessados em aprimorar a governança em gestão de projetos também podem utilizar os resultados deste trabalho.

\begin{abstract}
Ethics and social responsibility issues are accretive in the organization strategic agenda. Management mechanisms that aim links strategy and operations helps to insert those themes in the corporate culture. The OPM3, PMI's Organizational Project Management Maturity Model has the objective to help organizations to develop capabilities to align their strategic objectives with their operations through projects. In spite of OPM3's development was based in many researches, with contribution from more than 700 specialists and has actually a broadly utilization, it's not free of critics. The objective of this work is present existing critics to OPM3 and find new critics related to OPM3's redundancy. The redundancy investigation is treated through data modeling approach and the actual critics assessed thorugh a bibliographic revision. The results portray how to transform 456 of 586 OPM3's best practices in 38 and how to transform 120 of 151 questions from the OPM3's high-level assessment in 10 structured questions. The conclusion present a resume of critics that could be useful to whom that is involved in the use of OPM3, researches from maturity's model in Project Management and for a future revision of OPM3.
\end{abstract}

Key-words: project management maturity model, OPM3 critics, strategic and operations link.

\title{
Referências
}

Alleman, G. Some Thoughts on Project Management Organizational Maturity Models. In: PM FORUM, PM World Today, 2005. Disponível $<$ http://www.pmforum.org/viewpoints/2005/0102stopmomm.htm>. Acesso em: Julho 2005

BOUER, R. \& CARVALHO, M.M. Metodologia singular de gestão de projetos: condição suficiente para a maturidade em gestão de projetos? Revista Produção, v.15, n.3, p.347-361, Set./Dez, 2005.

CMM-I. Capability Maturity Model Integration - version 1.1 - for System Engineering and Software Engineering - CMMI-SE/SW/IPPD/SS, V1.1 continuous representation CMU/SEI-2002-TR-011 ESCTR-2002. Disponível em: <www.sei.cmu.edu>. Acesso em: mar, 2002.

COOKE-DAVIES T.J.; SCHLICHTER F. J.; BREDILlET,C. Beyond the PMBOK Guide. PMI Annual Seminars and Symposium 2001. Nashville T.S., 2001.

COOKE-DAVIES T.J; ARZYMANOW A. The maturity of project management in different industries: An investigation into variations between project management models. International Journal of Project Management, n. 21, p. 471-478, 2003.

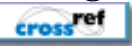


CONSTRUCTION INDUSTRY INSTITUTE. Cost-trust relationship. USA: Construction Industry Institute, 1993.

DEMING,W.E. Out of the Crisis. MIT Press International, Cambridge, Mass.,1982.

HUMPHREY, W.S. Managing the software process. Reading, Addison-Wesley (SEI series in software engineering), 1989.

HILLSON, D. Assessing Organizational Project Management Capability. Journal of Facilities Management, v.2. Iss 3, p.298, London, 2003.

JUGDEV, K.; THOMAS, J. Project Management Maturity Models: The Silver Bullets of Competitive Advantage? Project Management Journal, n. 33, p.4-14.; Dec. 2002.

KENNETH, 2004 - Organizational Project Management Maturity Model. Project Management Journal; 35,Vol 1, p.59, Apr 2004.

KERZNER H. Strategic planning for project management maturity model. New York: John Wiley \& Sons, 2001.

MORRIS, P.W.G. Researching the unanswered questions of Project Management. In: Proceedings of the PMI Research Conference 2000, Paris, 2000.

PMI, PROJECT MANAGEMENT INSTITUTE. A Guide to the Project Management Body of Knowledge (PMBoK). 3rd edition. Pennsylvania, USA: Project Management Institute Inc, 2004.

PMI, PROJECT MANAGEMENT INSTITUTE. Organisational Project Management Maturity Model (OPM3). Maryland, USA: Project Management Institute Inc; 2003.

RABECHINI, R.J.; CARVALHO, M.M. Perfil das Competências em Equipes de Projetos, RAE-eletrônica, v.2, n.1, Jan-Jun/2003.

SAWYER,P.; SOMMERVILLE I.; VILLER, S. Capture the benefits of Requirements Engineering. IEEE Software, v.16,n.2, p.78-85; 1999.

cross ref

SCHLICHTER, J.; FRIEDRICH, R.; HAECHK,B. The history of OPM3. In: PMI's Global Congress Europe 2003, Den Haag- Netherlands. Disponível em: $<$ http://www.pmforum.org/libray/papers/thhistoriyofopm3.htm>, Acesso em: Julho 2006.

SETZER V.W., SILVA, F.S.C. Banco de Dados: Aprenda o que são, Melhore seu conhecimento, Construa o seu. 1 ed São Paulo: Editora Edgard Blücher, 2005.

SOLER, A.M. OPM3: A contribuição PMI para a maturidade em gestão de projetos. Revista Mundo PM, n. III - Ano I, 2005.

SOMMERVILLE I.; LANGFORD, V. Multivariate influences on the people side of projects: stress and conflict. International Journal of Project Management, n.12, p.4, 1992.

SOMMERVILLE I.; RANSOM, J. An Empirical Study of Industrial Requirements Engineering Process Assessment and Improvement. In: ACM Transactions on Software Engineering and Methodology, Computer Department, Lancaster University, v. 14, n.1,p. 85-117, Jan. 2005.

\section{Dados dos autores:}

Nemer Alberto Zaguir

Escola Politécnica da Universidade de São Paulo / Oracle do Brasil

Departamento: Engenharia Naval e Oceânica / Consultoria

Endereço: Av. José Bonifácio, 1391, Ap. 64 B Campinas, SP, Brasil, CEP 13091-240

Telefones para contato: (11) 8245-1495 
e-mail:nemer.zaguir@oracle.com;nemer.zaguir@poli.usp.br

Dr. Marcelo Ramos Martins

Escola Politécnica da Universidade de São Paulo

Departamento: Departamento de Engenharia Naval e Oceânica

Função ou cargo ocupado: Professor

Endereço: Av. Prof. Mello Moraes, 2231, Cid. Universitária, São Paulo, SP, Brasil, CEP05508-900.

Telefones para contato: (11) 3091-5350

e-mail: rmrmartin@usp.br

Recebido para publicação em: 21/11/2006

Aceito para publicação em: 03/03/2007 